\title{
La génétique des lactobacilles, leuconostocs et pédiocoques : criblage, sélection et construction de mutants
}

\begin{abstract}
B Kammerer
Laboratoire de microbiologie et de génétique de l'université Louis-Pasteur, URA CNRS n'D1481 "dynamique, évolution et expression de génomes de micro-organismes", Institut de botanique, 28, rue Goethe, 67083 Strasbourg cedex, France
\end{abstract}

\begin{abstract}
Résumé - Les organismes vivants dont l'analyse génétique est la plus approfondie sont les mieux connus et les plus aisément modifiables. L'analyse des mutants apporte des informations essentielles sur la fonction des gènes mutés, leur organisation et leur régulation. L'absence de système génétique chez la plupart des bactéries lactiques restreint la compréhension de leur fonctionnement et la possibilité de les «améliorer». En principe, quelques étapes sont nécessaires pour obtenir des mutants : mutagenèse, expression, enrichissement, puis détection, dans la population mutagenéisée, des clones du phénotype recherché. Les premières étapes n'ont été que très rarement l'objet d'études systématiques chez les bactéries lactiques. Quelques méthodes plus ou moins astucieuses de criblage ou de sélection ont été mises au point pour détecter des mutants dans des systèmes particuliers aux bactéries lactiques (fermentation malolactique, utilisation des sucres, peptidases, production de polymères). Certaines parties du métabolisme ont fait l'objet d'études approfondies avec un grand nombre de mutants. C'est le cas des voies de biosynthèse des acides aminés que les bactéries lactiques exigent souvent pour leur croissance. Le groupe de Morishita a obtenu et analysé de nombreux mutants méiotrophes (capables de synthétiser un composé exigé par la souche sauvage, donc indépendants de ce composé), principalement chez les lactobacilles, ce qui lui a permis de montrer que dans un grand nombre de cas ces voies sont "presque" fonctionnelles. Par ailleurs, nous avons étudié des dizaines de mutants touchés dans le métabolisme des pyrimidines chez Lactobacillus plantarum CCM 1904, une souche prototrophe pour l'uracile ; la régulation en est originale puisqu'elle dépend, entre autre, de la nature aérobie, ou non, du milieu. Depuis quelques années des mutants parfaitement définis au niveau moléculaire ont pu être construits chez les lactobacilles, sans trace de vecteur ou de marqueur, ce qui devrait permettre, dans certains cas, leur utilisation sans risque dans des écosystèmes ouverts. Cette possibilité ouvre également la voie, en compensant l'absence de système de transfert, à des analyses génétiques beaucoup plus poussées.
\end{abstract}

\section{génétique / mutant / Lactobacillus / Leuconostoc / Pediococcus}

Summary - Genetics of lactobacilli, leuconostocs et pediococci : screening, selection and construction of mutants. There is no genetic system for the majority of lactic acid bacteria species. The first steps of mutant isolation (mutagenic treatment, enrichment) are very rarely studied. Some screening or selection methods were designed to obtain mutants in pathways of interest within lactic 
acid bacteria such as: malolactic fermentation, sugar utilization, peptidases, glucan production, etc. More precisely, two pathways are studied with a great number of mutants. Morishita has shown that nutritional requirements in multiple auxotrophic lactic acid bacteria are often the consequence of minor genetic lesions repairable by single-step mutations. We have studied pyrimidine metabolism in Lactobacillus plantarum CCM 1904, a prototrophic strain, by analysing more than 50 mutants: the regulation differs in aerobiosis and in anaerobiosis. In recent years, lactobacilli mutants, perfectly defined at the molecular level, had been constructed without vector sequences in the final structure. It would be possible to release these organisms in an open system and use them in genetic analysis, thus overcoming the absence of natural genetic transfer.

\section{genetics / mutant / Lactobacillus / Leuconostoc / Pediococcus}

\section{INTRODUCTION}

"When you have a mutant you are better off than when you don't." (S Luria). On ne dispose à l'heure actuelle que d'informations limitées sur la structure, l'organisation et l'expression des génomes des lactobacilles, leuconostocs et pédiocoques. Très peu de mutants ont été recherchés; les techniques classiques de transfert de gènes chromosomiques chez les bactéries (transformation, transduction, conjugaison) n'ont pas été développées dans ces trois genres. Les techniques d'analyse génétique seraient pourtant d'une grande utilité, aussi bien du point de vue fondamental que médical ou économique. La mise en place d'un système génétique efficace est un travail de longue haleine qui n'a été réalisé que pour quelques espèces bactériennes (Escherichia coli, Salmonella typhimurium, Bacillus subtilis), celles qui, actuellement, servent d'espèces modèle. L'analyse par des moyens génétiques d'une propriété d'un organisme suppose, en premier lieu, l'obtention de mutants, puis, l'étude comparée des phénotypes des mutants et de la souche sauvage, et, si l'on dispose des techniques appropriées, l'étude des rapports entre les différentes mutations (complémentation, dominance, épistasie).

Cet article présente une revue des mutants (de gènes chromosomiques) dans les genres Lactobacillus, Leuconostoc et
Pediococcus obtenus par des moyens classiques ou par intégration chromosomique d'une construction in vitro. Cette revue n'est certainement pas exhaustive mais espère montrer que l'obtention de quelques mutants, même en l'absence d'une génétique sophistiquée, permet souvent de résoudre des problèmes d'un abord beaucoup plus difficile avec d'autres techniques. $\mathrm{Ne}$ seront pas traités les variants génétiquement mal définis bien que pratiquement très utiles (résistants obtenus par passages successifs dans des concentrations croissantes de l'inhibiteur, surproducteurs obtenus par plusieurs cycles de mutagenèsecriblage).

\section{TECHNIQUES UTILISÉES}

Un récapitulatif des techniques de mutagenèse employées par les différents laboratoires est présenté dans le tableau I. La très grande hétérogénéité des protocoles s'explique par la grande variété de souches et, semble-t-il, l'absence d'optimisation. Rodriguez-Quiñones et al $(1982,1984)$ ont étudié l'effet de la concentration en $\mathrm{N}$ méthyl-N'-nitro-N-nitrosoguanidine (MNNG), de la composition du tampon, de la durée du traitement et de la température sur l'efficacité de la mutagenèse chez Lactobacillus plantarum LS7. Masson et al (1994) ont effectué le même travail pour $L$ plantarum 
CCM 1904 et mis au point une technique d'enrichissement en mutants par l'ampicilline. Bien que concernant deux souches de la même espèce, ces deux méthodes optimales sont dissemblables.

\section{MUTANTS MÉIOTROPHES}

Morishita et al en 1974 entreprennent une analyse génétique systématique des exigences nutritionnelles des lactobacilles. Après mutagenèse d'une souche sauvage de Lactobacillus casei à la N-méthyl-N'nitro-N-nitrosoguanidine (MNNG), à la 2amino-purine (2AP) ou aux rayons ultra-violets (UV), ils sélectionnent les mutants méiotrophes, c'est-à-dire capables de pousser sur un milieu minimum synthétique dépourvu de l'un ou l'autre des composés normalement exigés. Parmi les douze acides aminés exigés par le sauvage sept peuvent être synthétisés par l'un ou l'autre des mutants ; c'est le cas également de trois vitamines sur quatre. Cela suggère que les nombreuses exigences nutritionnelles de L casei sont le plus souvent dues à des petites lésions plutôt qu'à de grandes délétions dans les gènes impliqués dans les voies de biosynthèse.

Parmi une collection de mutants (spontanés ou induits par la 2-amino-purine) de L casei résistants à la rifampicine, Morishita et Yura (1976) constatent que la moitié présente, par rapport à la souche sauvage de départ, une exigence nutritionnelle pour la glutamine et que quelques uns n'exigent plus l'aspartate. La moitié des mutants exigeant la glutamine sont également incapables d'utiliser le maltose ou le mannose comme seule source de carbone. II est possible, à partir de ces mutants, de sélectionner des révertants qui perdent simultanément leur exigence pour la glutamine et leur résistance à la rifampicine. L'analyse enzymatique de l'un de ces révertants montre que c'est bien la résistance ou la sensibi- lité de l'ARN polymérase à la rifampicine qui est en cause et qu'une modification de l'ARN polymérase affecte le métabolisme de la glutamine.

Pour voir si la nature et l'étendue des lésions génétiques dans les voies de biosynthèse des lactobacilles dépendent de leurs habitats d'origine, Morishita et al (1981) ont entrepris une étude systématique des mutations méiotrophes pour les acides aminés chez quatre espèces. Dans chaque souche des mutants méiotrophes ont été obtenus pour de nombreux acides aminés, principalement après mutagenèse à la MNNG (5 sur 7 pour $L$ plantarum, $6 / 10$ pour $L$ casei, $10 / 14$ pour $L$ helveticus et $4 / 15$ pour $L$ acidophilus). Certains de ces mutants sont thermosensibles (Met et Trp chez L plantarum). Certaines espèces qui présentent les mêmes exigences diffèrent beaucoup dans leurs possibilités de "réparation" ( $L$ helveticus et $L$ acidophilus). Les voies de biosynthèse peuvent être réparties en cinq groupes: (I) celles qui sont actives chez tous les lactobacilles (Ala, Asp, Gin et Gly) ; (II) celles qui sont actives ou inactives mais "réparables" chez tous les lactobacilles (lle, Leu, Lys, Ser et Tyr) ; (III) celles qui sont actives ou inactives mais "réparables" chez tous les lactobacilles sauf $L$ acidophilus (Asp, Cys, Pro et Phe) ; (IV) celles qui ne sont pas "réparables" chez $L$ acidophilus et au moins une autre espèce (Arg, His, Met, Thr, Trp et $\mathrm{Val}$ ) ; et ( $\mathrm{V}$ ) celle qui n'est jamais active (Glu). Morishita et Yajima (1995) expliquent l'incapacité des lactobacilles à synthétiser le glutamate en montrant que le cycle de Krebs n'est pas fonctionnel: l'absence d'isocitrate déshydrogénase bloque la branche oxydative; la branche réductrice va jusqu'au succinate (peut-être avec un rôle énergétique de la fumarate réductase). II n'y a pas de corrélation entre les exigences nutritionnelles et le nombre d'étapes enzymatiques dans chaque voie de biosynthèse. En revanche, la corrélation est nette entre l'étendue des lésions géné- 
Tableau I. Paramètres utilisés pour les mutagenèses.

Parameters used in mutageneses.

$\begin{array}{llll}\text { Mutagène Souche } & \text { Tampon } & \begin{array}{l}\text { Concen- } \\ \text { tration }\end{array} & \begin{array}{l}\text { Temp } \\ \left({ }^{\circ} \mathrm{C}\right)\end{array}\end{array}$ Durée $\quad$ Rérence

\section{AP Lb caseiS1}

2AP Lb caseiS1

$\begin{array}{ll}\text { EMS } & \text { Lb acidophilus ATCC } 11506 \\ \text { EMS } & \text { Lb casei ATCC } 7469 \\ \text { EMS } & \text { Lb helveticus ATCC } 15009 \\ \text { EMS } & \text { Lb plantarum ATCC } 8014 \\ \text { EMS } & \text { Lc mesenteroides NRRL B-1355 } \\ \text { EMS } & \text { Lc mesenteroides NRRL B-512F } \\ \text { EMS } & \text { Pc acidilactici ATCC } 8042\end{array}$

EMS

$\mathrm{HNO}_{2} \quad$ Lactobacillus sp 89

$\begin{array}{ll}\text { MNNG } & \text { Lactobacillus 30a } \\ \text { MNNG } & \text { Lb acidophilus ATCC } 11506 \\ \text { MNNG } & \text { Lb bulgaricus CNRZ } 397 \\ \text { MNNG } & \text { Lb casei ATCC } 7469 \\ \text { MNNG } & \text { Lb casei ATCC } 7469 \\ \text { MNNG } & \text { Lb casei } 11 \\ \text { MNNG } & \text { Lb casei } 1\end{array}$

milieu de Rogosa

milieu de Rogosa

milieu de Rogosa, $\mathrm{pH} 7,2$

milieu de Rogosa, $\mathrm{pH} 7,2$

milieu de Rogosa, $\mathrm{pH} 7,2$

milieu de Rogosa, $\mathrm{pH} 7,2$

$10 \mathrm{ml}$ Lawford + Glc 1\%

phosphate de $\mathrm{Na} 100 \mathrm{mmol} / \mathrm{pH} 7$

milieu de Rogosa, $\mathrm{pH} 6,8$

acétate de $\mathrm{Na} 0,1 \mathrm{~mol} / / \mathrm{pH} 4,5$

milieu de Vogel-Bonner

phosphate $0,05 \mathrm{~mol} / / \mathrm{pH} 7$

phosphate $0,05 \mathrm{~mol} / \mathrm{l} \mathrm{pH} 7$

acétate $0,2 \mathrm{~mol} / / \mathrm{pH} 5$

citrate de sodium $0,1 \mathrm{~mol} / / \mathrm{pH} 5,5$

\section{$50 \mu \mathrm{g} / \mathrm{ml}$}

$50 \mu \mathrm{g} / \mathrm{ml}$

37

37

$24 \mathrm{~h}$

une nuit

$2 \%$

$2 \%$

$2 \%$

$2 \%$

$+0,4 \mathrm{ml}$

$31 \mathrm{mg} / \mathrm{ml}$

$2 \%$

37
37
37
37
30
28
37

$30 \mathrm{~min}$

$45 \mathrm{~min}$

$30 \mathrm{~min}$

$45 \mathrm{~min}$

$48 \mathrm{~h}$

$60 \mathrm{~min}$

$30 \mathrm{~min}$

37

$1 \% *$

$200 \mu \mathrm{g} / \mathrm{m}$

$100 \mu \mathrm{g} / \mathrm{ml}$

$50 \mu \mathrm{g} / \mathrm{ml}$

$100 \mu \mathrm{g} / \mathrm{ml}$

$100 \mu \mathrm{g} / \mathrm{ml}$

$700 \mu \mathrm{g} / \mathrm{ml}$

$100 \mu \mathrm{g} / \mathrm{ml}$
$30 \mathrm{~min}$

$70 \mathrm{~min}$
$30 \mathrm{~min}$

$60 \mathrm{~min}$

$3 \mathrm{~h}$

$30 \mathrm{~min}$
Morishita et Yura, 1976

Morishita et al, 1974

Morishita et al, 1981

Morishita et al, 1981

Morishita et al, 1981

Morishita et al, 1981

Smith et al, 1994

Kim et Robyt, 1994

Deguchi et Morishita, 1992

Chagnaud et al, 1990

Recsei et Snell, 1972

Morishita et al, 1981

Atlan et al, 1989

Morishita et al, 1981

Ntamere et al, 1987

Morishita et al, 1974

Shimizu-Kadota et Sakurai, 1982 
MNNG

MNNG

MNNG

MNNG

MNNG

MNNG

MNNG

MNNG

MNNG

MNNG

\section{Lb helveticus ATCC 15009 \\ Lb plantarum ATCC 8014 \\ Lb plantarum ATCC 8014 \\ Lb plantarum ATCC 8014 \\ Lb plantarum LS7 \\ Lb plantarum WSO}

Lc mesenteroides NRRL B-512F

Lc oenos

Pc acidilactici ATCC 8042

PC cerevisiae ATCC 8081

\section{Lb acidophilus ATCC 11506}

Lb casei ATCC 7469

Lb casei 1

Lb helveticus ATCC 15009

Lb plantarum ATCC 8014

Lc mesenteroides NRRL B-1355

Lc mesenteroides NRRL B-1355

Lc oenos

PC acidilactici ATCC 8042 phosphate $0,05 \mathrm{~mol} / \mathrm{l} \mathrm{pH} 7$

phosphate de $\mathrm{K} 100 \mathrm{mmol} / \mathrm{pH} 7$

phosphate $0,05 \mathrm{~mol} / \mathrm{l} \mathrm{pH} 7$

phosphate de $\mathrm{Na} 20 \mathrm{mmol} / / \mathrm{pH} 6$

Tris-maléate $0,2 \mathrm{~mol} / / \mathrm{pH} 5,5$

salin

phosphate $50 \mathrm{mmol} / \mathrm{l} \mathrm{H} 6,2$

salin

phosphate $0,05 \mathrm{mmol} / / \mathrm{pH} 7$

Tris-maléate $0,1 \mathrm{~mol} / / \mathrm{pH} 6$

$\begin{array}{lll}100 \mu \mathrm{g} / \mathrm{ml} & 37 & 30 \mathrm{~min} \\ 170 \mu \mathrm{g} / \mathrm{ml} & 30 & 15 \mathrm{~min} \\ 100 \mu \mathrm{g} / \mathrm{ml} & 37 & 30 \mathrm{~min} \\ 200 \mu \mathrm{g} / \mathrm{ml} & 37 & 30 \mathrm{~min} \\ 500 \mu \mathrm{g} / \mathrm{ml} & 28 & 30 \mathrm{~min} \\ 500 \mu \mathrm{g} / \mathrm{ml} & 30 & 90 \mathrm{~min} \\ 500 \mu \mathrm{g} / \mathrm{ml} & 30 & 60 \mathrm{~min} \\ 500 \mu \mathrm{g} / \mathrm{ml} & 30 & 90 \mathrm{~min} \\ 100 \mu \mathrm{g} / \mathrm{ml} & 37 & 30 \mathrm{~min} \\ 100 \mu \mathrm{g} / \mathrm{ml} & 37 & 30 \mathrm{~min}\end{array}$

salin

salin

salin?

salin

salin

$\mathrm{MgSO}_{4} 10 \mathrm{mmol} / \mathrm{l}$

$\mathrm{MgSO}_{4} 0,1 \mathrm{~mol} / \mathrm{l}$ ou $\mathrm{NaCl} 0,85 \%$

eau

salin
$15 \mathrm{~W}$ à $50 \mathrm{~cm}$

$15 \mathrm{~W}$ à $50 \mathrm{~cm}$

$15 \mathrm{~W}$ à $60 \mathrm{~cm}$

$15 \mathrm{~W}$ à $50 \mathrm{~cm}$

$15 \mathrm{~W}$ à $50 \mathrm{~cm}$

$330 \mathrm{~W} / \mathrm{cm}^{2}$

$120000 \mathrm{erg} / \mathrm{cm}^{2}$

$845 \mathrm{erg} / \mathrm{mm}^{2}$

$15 \mathrm{~W}$ à $50 \mathrm{~cm}$
$60 \mathrm{~s}$

$30 \mathrm{~s}$

$60 \mathrm{~s}$

$60 \mathrm{~s}$

$30 \mathrm{~s}$

$10-30 \mathrm{~s}$

$0,1 \%$ *

$90 \mathrm{~s}$
Morishita et al, 1981

Masson et al, 1994

Morishita et al, 1981

Tamura et Matsushita, 1992

Rodríguez-Quiñones et al, 1984

Daeschel et al, 1984

Mizutani et al, 1994

Lautensach et Subden, 1987

Deguchi et Morishita, 1992

Widdowson et White, 1976

Morishita et al, 1981

Morishita et al, 1981

Morishita et al, 1974

Morishita et al, 1981

Morishita et al, 1981

Leathers et al, 1995

Smith et al, 1994

Zúñiga et al, 1994

Deguchi et Morishita, 1992

2AP : 2-amino-purine ; EMS : éthyl-méthane-sulfonate ; $\mathrm{HNO}_{2}$ : acide nitreux ; MNNG : N-méthyl-N'-nitro-N-nitrosoguanidine ; UV : ultra-violet. * : durée de traitement nécessaire pour obtenir ce pourcentage de survie.

2AP: 2-amino-purine; EMS: ethyl-methane-sulfonate; $\mathrm{HNO}_{2}$ : nitrous acid; MNNG: N-methyl-N'-nitro-N-nitrosoguanidine; UV: ultra-violet. * : length of treatment necessary to obtain this level of survival. 
tiques et l'impossibilité d'utiliser des précurseurs de l'acide aminé exigé.

II ressort de ce travail que la plupart des gènes de biosynthèse sont présents chez les lactobacilles mais souvent avec quelques petites lésions qui ne permettent pas la synthèse d'enzymes fonctionnelles. Pour certaines souches et certaines voies les lésions sont trop importantes pour permettre l'obtention de mutants méiotrophes en une seule étape. Tout cela semble bien corrélé avec les caractéristiques écologiques des souches étudiées : celles qui trouvent toujours dans leur environnement un acide aminé donné vont perdre définitivement la capacité à le synthétiser ; celles qui parfois se trouvent carencées doivent maintenir un génome à peu près intact.

Deguchi et Morishita (1992) ont étendu ce travail à trois autres genres de bactéries lactiques: Lactococcus lactis, Enterococcus faecium et Pediococcus acidilactici. Contrairement aux lactobacilles certains mutants méiotrophes peuvent être obtenus spontanément mais ils se révèlent instables. Des mutants méiotrophes ont pu être obtenus pour la moitié des acides aminés exigés par chacune des souches. Une classification des voies de biosynthèse du même type que celle obtenue avec les lactobacilles peut être réalisée. Quatre acides aminés ne sont jamais synthétisés (His, Met, Val et Glu). Chez $E$ faecium et $P$ acidilactici une seule mutation peut conduire à la méiotrophie pour plusieurs acides aminés (jusqu'à cinq) ; le nombre et le type d'acides aminés pour lesquels les souches deviennent méiotrophe varient beaucoup et dépendent de la souche de départ et du phénotype sélectionné. Plus de la moitié des mutants d'E faecium méiotrophes pour plusieurs acides aminés (sélectionnés sur leur capacité à synthétiser la tyrosine) sont plus sensibles à la rifampicine que la souche sauvage. La recherche, parmi ces mutants, de révertants sur la base d'une résistance normale à la rifampicine conduit à l'obtention de souches beaucoup plus résistantes que la souche sauvage et incapables, à nouveau, de synthétiser une partie des acides aminés pour lesquels elles étaient méiotrophes. On peut donc en conclure que l'acquisition par une mutation unique, chez E faecium, de la capacité à synthétiser plusieurs acides aminés est liée à une altération de l'ARN polymérase qui pourrait permettre, par exemple, la synthèse d'une transaminase commune à plusieurs voies de biosynthèse.

\section{LA FERMENTATION MALOLACTIQUE}

Daeschel et al (1984) ont criblé des mutants de Lactobacillus plantarum déficients en malate décarboxylase en utilisant un milieu différentiel qui contient du vert de bromocrésol (indicateur de $\mathrm{pH}$ de 3,8 à 5,4 ), une faible concentration de glucose $(0,5 \%)$ comme source d'énergie et une forte concentration de malate $(2 \%)$. Sur ce milieu une souche qui décarboxyle le malate maintient un $\mathrm{pH}$ neutre (colonies bleu foncé) ; une souche qui ne décarboxyle pas le malate ne peut pas compenser l'acidification due au glucose transformé en acide lactique (colonie jaune-vert). Cette technique est d'usage générale mais doit être adaptée pour chaque espèce (Streptococcus lactis : Renault et Heslot, 1987 ; Leuconostoc oenos: Cavin et al, 1989).

Lautensach et Subden (1987) utilisent le système de criblage de Daeschel et al (1984) pour obtenir une vingtaine de mutants de Leuconostoc oenos déficients en activité malolactique. Plusieurs loci peuvent être impliqués : transport du malate, induction de l'enzyme, activité de l'enzyme... Les mutants obtenus ont des profils variés en terme de croissance ou d'activité malolactique selon le $\mathrm{pH}$ et d'utilisation du malate. Tous les mutants de désacidification obtenus ont perdu leur activité malolactique ce qui montre que l'enzyme 
malolactique est la responsable principale de la désacidification. Mais le crible utilisé ne permet pas d'écarter d'autre rôle physiologique de la fermentation malolactique.

Chagnaud et al (1990) utilisent une méthode de criblage beaucoup plus lourde (croissance en MRS avec ou sans malate, transfert dans une solution de malate avec un indicateur de $\mathrm{pH}$, mesure de l'absorbance après $24 \mathrm{~h}$ ) mais qui permet de différencier les mutants déficients, les mutants hyperproducteurs et les mutants constitutifs.

Zúñiga et al (1994) ont mis au point un milieu sélectif pour isoler des mutants malolactiques de Leuconostoc oenos en utilisant le fait que la croissance peut être inhibée par l'augmentation de $\mathrm{pH}$ due à la fermentation malolactique si le $\mathrm{pH}$ initial est suffisamment élevé ; les mutants malolactiques ne feront qu'acidifier le milieu sans inhiber leur propre croissance. L'équilibre entre les concentrations en sucres et en acide malique ainsi que le $\mathrm{pH}$ initial sont très importants pour le fonctionnement de cette méthode de sélection ; $40 \%$ des colonies qui poussent sont effectivement déficientes en enzyme malolactique; d'autres sont des souches qui acidifient plus efficacement le milieu.

La fermentation malolactique n'est pas le seul mécanisme de diminution de l'acidité. Pour étudier l'hypothèse que les aminoacides décarboxylases inductibles permettent de lutter contre une acidité trop forte, Recsei et Snell (1972) effectuent une mutagenèse à la MNNG sur Lactobacillus 30a et repèrent les mutants sur un milieu à $\mathrm{pH} 5$ contenant de l'histidine et du rouge de méthyle; les colonies sauvages peuvent augmenter le $\mathrm{pH}$ et deviennent jaunes; les colonies déficientes en histidine décarboxylase sont incapables de remonter le $\mathrm{pH}$ et restent rouges. Des mesures de croissance, de variation de $\mathrm{pH}$ et d'activité permettent de montrer qu'effectivement l'histidine décarboxylase inductible permet une meilleure croissance lorsque le $\mathrm{pH}$ initial est bas ou lorsque le milieu permet une forte production d'acide lactique.

\section{PAROI ET TRANSPORT}

Ntamere et al (1987) ont obtenu cinq mutants de Lactobacillus casei dont l'acide lipotéichoïque ne contient pas d'ester de Dalanine, après mutagenèse à la MNNG et criblage des colonies par leur incapacité à incorporer de la D-alanine marquée. Par des analyses biochimiques et microscopiques, ils ont pu montrer que deux de ces mutants sont affectés dans la D-alanylation de l'acide lipotéichoïque; deux autres sont partiellement déficient dans l'enzyme d'activation de la $\mathrm{D}$-alanine; tous les mutants présentent une forme incurvée et des sites de séparation entre cellules anormaux. Dans l'ignorance du caractère isogénique ou non des souches, l'analyse des phénotypes est rendue délicate.

Atlan et al (1989, 1990) obtiennent des mutants déficients en aminopeptidase II par criblage par essai enzymatique sur boîte d'une population mutagenéisée de Lactobacillus bulgaricus en fonction de la capacité à hydrolyser l'alanyl- ou le leucyl-bêta-naphtylamide. L'analyse des activités peptidasiques de ces mutants dans différentes conditions de culture permet de conclure à l'existence de quatre aminopeptidases et d'une X-prolyl-dipeptidyl-aminopeptidase spécifique. Des mutants déficients en $X$ prolyl-dipeptidyl-aminopeptidase sont repérés par leur incapacité à hydrolyser la glycylprolyl-bêta-naphtylamide. Les mutations obtenues ont un effet pléiotrope sur les protéines de paroi. Les analyses effectuées sur les deux types de mutants obtenus montrent qu'il existe des systèmes de régulation communs pour la biosynthèse de l'aminopeptidase II, de I'X-prolyl-dipeptidylaminopeptidase et des protéinases de paroi.

Lactobacillus plantarum peut croître sur mélibiose à $30^{\circ} \mathrm{C}$ mais pas à $37^{\circ} \mathrm{C}$ alors 
qu'il croît sur galactose ou lactose aux deux températures. La physiologie du transport et de l'hydrolyse du mélibiose suggère que c'est le mécanisme d'induction du système de transport du mélibiose (mais pas de l'alpha-galactosidase) qui est thermosensible. Pour éviter les interférences entre les systèmes d'utilisation du lactose et du mélibiose, Tamura et Matsushita (1992) isolent un mutant déficient dans le transport du lactose qui leur permet de démontrer que le transport et l'hydrolyse du mélibiose sont soumis à des régulations différentes : le mécanisme d'induction du système de transport du mélibiose (mais pas de l'alphagalactosidase) est thermosensible alors que le système de transport lui-même ne l'est pas (c'est l'inverse chez Escherichia coll). Le système de transport du lactose n'est pas induit par le mélibiose (contrairement à celui d'E coli) bien qu'il le transporte à toutes les températures.

Veyrat et al (1994) isolent un mutant spontané résistant au 2-désoxy-D-glucose à partir d'un Lactobacillus casei curé. La comparaison des propriétés de croissance et de consommation de sucres entre la souche sauvage et ce mutant permet de montrer que la mutation touche un élément du complexe llABCman qui, dans la souche sauvage, est responsable de la forte répression par le glucose et le mannose des gènes d'utilisation du lactose et du ribose ; les gènes d'utilisation du galactose seraient soumis à un autre mécanisme de répression catabolique.

\section{PRODUCTION DE POLYSACCHARIDES}

Les glucane-sucrases de Leuconostoc mesenteroides ne sont produites qu'après induction par le saccharose mais le saccharose est alors transformé en dextranes, ce qui perturbe l'étude des enzymes et du mécanisme de synthèse des différents types de dextranes. Pour repérer des mutants de
L mesenteroides constitutifs pour la glucane-sucrase Kim et Robyt (1994, 1995) effectuent un dosage enzymatique pour chacun des clones résultant de la mutagenèse à l'éthyl-méthane-sulfonate (EMS). Ils font trois ou quatre cycles de mutagenèse pour obtenir des souches hyperproductrices d'enzyme ou des souches ne produisant qu'un seul type de dextrane-sucrase.

Par mutagenèse UV ou EMS puis criblage des colonies sur des critères morphologiques Smith et al (1994) obtiennent des mutants touchés dans la production de l'une ou l'autre des trois principales glucosyltransférases et des glucanes (dextranes ou alternanes) de $L$ mesenteroides NRRL B1355. Ces modifications biochimiques expliquent les différences de morphologie des colonies et les propriétés des cultures liquides.

La synthèse enzymatique du dextrane présente plusieurs avantages par rapport à la synthèse par les microorganismes entiers ; mais il faut alors une méthode de production de dextrane-sucrase. La synthèse de la dextrane-sucrase est induite par le saccharose mais le saccharose est transformé en dextrane, ce qui perturbe la purification de l'enzyme. Pour produire cette enzyme en l'absence de saccharose Mizutani et al (1994) recherchent des mutants de $L$ mesenteroides NRRL B-512F constitutifs pour la production de dextranesucrase. La population mutagenéisée est étalée sur MRS ; $48 \mathrm{~h}$ plus tard la boîte est recouverte de gélose molle contenant de la tétracycline (pour empêcher la synthèse de dextrane-sucrase) et du saccharose. Dans ces conditions le dextrane est synthétisé uniquement par les colonies qui produisent la dextrane-sucrase de façon constitutive.

À partir d'une souche de $L$ mesenteroides NRRL B-1355 qui produit de l'alternane et du dextrane (mélange difficile à séparer), Leathers et al (1995) recherchent des mutants qui produisent une forte proportion d'alternane. Après une mutagenèse 
aux UV 5280 clones survivants sont transférés dans des plaques de microtitration dans un milieu au saccharose $; 48 \mathrm{~h}$ plus tard est ajoutée de la dextranase puis est fait un traitement qui révèle les sucres réducteurs. Les puits dont la coloration est située entre celle du témoin positif (sauvage avec dextranase) et celle du témoin négatif (sauvage sans dextranase) correspondent à des faibles producteurs de dextrane donc à d'éventuels bons producteurs relatifs d'alternane.

\section{MÉTABOLISME DES PYRIMIDINES CHEZ LACTOBACILLUS PLANTARUM}

Masson et al $(1992,1994)$ ont optimisé la mutagenèse et l'enrichissement en mutants chez Lactobacillus plantarum CCM 1904 et obtenu ainsi 280 mutants impliqués dans le métabolisme des pyrimidines. L'analyse physiologique de 56 de ces mutants (croissance et activités enzymatiques en fonction du milieu et des conditions physico-chimiques, en présence de divers intermédiaires de ce métabolisme, d'analogues ou d'inhibiteurs) a été effectuée. Les activités des enzymes impliqués ont été déterminées dans les mutants auxotrophes et bradytrophes, ce qui a permis de définir les six loci impliqués dans la biosynthèse ( $p y r A$, $B, C, D, E, F$. Les mesures de croissance et l'effet des analogues de pyrimidines sur les souches mutantes et sauvage ont permis de définir d'autres loci ( $p d p$, upp, $u d k$, tdk, thy $A, p y r G$ ) et de montrer que le métabolisme des pyrimidines chez $L$ plantarum est classique, à l'exception de la non utilisation de la cytosine. Par contre, la régulation de la biosynthèse des pyrimidines chez $L$ plantarum n'est pas classique et dépend de la présence de l'arginine ou de l'oxygène dans le milieu de culture : la croissance de la souche sauvage est inhibée par l'uracile, l'uridine ou la désoxyuridine en aérobiose mais pas en anaérobiose ; cette inhibition de croissance est supprimée par la présence d'arginine ou de citrulline. Une sensibilité particulière à l'oxygène ou au $\mathrm{CO}_{2}$ de la carbamylphosphate synthétase spécifique de la biosynthèse de l'arginine peut expliquer ce comportement.

\section{MUTATIONS CONDITIONNELLES}

La fermentation lactique de lait écrémé par Lactobacillus casei (souche S-1) est parfois contaminée par un phage virulent (FSV) dont la souche sauvage (FSW) est un phage tempéré qui lysogénise la souche $\mathrm{S}-1$. Shimizu-Kadota et Sakurai (1982) font I'hypothèse que le curage de la souche S-1 permettrait d'éviter l'apparition de phages virulents au cours de la production. Le curage peut se faire par sélection d'une souche sensible parmi les survivants après induction du phage. Dans le cas particulier les traitements habituels (UV, mitomycine C) ne provoquent pas d'induction. Les auteurs recherchent donc des mutants thermo-inductibles : parmi une collection de mutants thermosensibles ils recherchent ceux qui ont une meilleure productivité en phages après un traitement thermique. Les révertants spontanés ayant exactement les caractéristiques du sauvage la mutation est vraisemblablement unique. Un cinquième des survivants à un traitement thermique se révèlent sensibles au phage $F S W$ et ne produire de phages à aucune température. En construisant toutes les combinaisons de souches lysogènes on peut montrer que la mutation responsable de la thermo-inductibilité et de la thermosensibilité de l'hôte est localisée sur le prophage : la souche curée, malgré la mutagenèse, a donc exactement les mêmes caractéristiques que la souche de départ mais sans les accidents de fermentation dus au phage. A ma connaissance, il s'agit du seul exemple du criblage d'une collection de mutants thermosensibles chez les lactobacilles. 


\section{ANALYSE MOLÉCULAIRE DES MUTATIONS}

La nature moléculaire des mutations est rarement connue. Pour étudier la stabilité génétique de Lactobacillus bulgaricus, Mollet et Delley (1990) criblent sur X-Gal une centaine de mutants spontanés déficients en bêta-galactosidase. Dix de ces mutations sont des délétions localisées principalement dans une petite zone du gène et résultant le plus souvent de la présence de courtes séquences directement répétées de 13 ou 14 paires de bases séparées par 30 à 150 paires de bases. L'un de ces mutants de délétion peut recouvrer une activité bêtagalactosidase (Mollet et Delley, 1991). Dans les quatre cas indépendants étudiés, la réversion consiste en la duplication d'un fragment de 72 paires de bases compris entre deux séquences directement répétées de 13 paires de bases adjacentes aux deux séquences directement répétées qui ont permis la délétion initiale. (Sauvage initial : rép. I - fragment I - rép. I - rép. II - fragment II - rép. II ; mutant : rép. I - rép. II fragment II - rép. II ; révertant : rép. I - rép.

Fig 1. Schéma de principe de l'intégration-excision. Première étape : construction de la mutation sur un vecteur plasmidique et transformation de la souche à modifier; deuxième étape : le plasmide est intégré au chromosome par recombinaison homologue (ici à gauche de la mutation); troisième étape : un second événement de recombinaison a lieu soit à droite de la mutation (comme indiqué), ce qui l'intègre dans le chromosome, soit à gauche ce qui reconstitue l'état initial.

General strategy for integration-excision. First step: construction of the mutation on a plasmid vector and transformation of the strain to be modified; second step: the whole plasmid is integrated into the chromosome by homologous recombination (here on the left side of the mutation); third step: subsequent homologous recombination can occur on the right side of the mutation (which is integrated in the chromosome, as indicated), or on the left side (the chromosome is left unmodified).
II - fragment II - rép. II - fragment II - rép. II.) Les souches révertantes peuvent facilement perdre leur phénotype $\mathrm{Lac}^{+}$par délétion d'un exemplaire du fragment dupliqué. La délétion d'un fragment de 10 acides aminés suivie de la duplication d'un fragment adjacent de 24 acides aminés conduit donc à une protéine qui présente les mêmes caractéristiques enzymatiques mais qui est beaucoup moins stable.

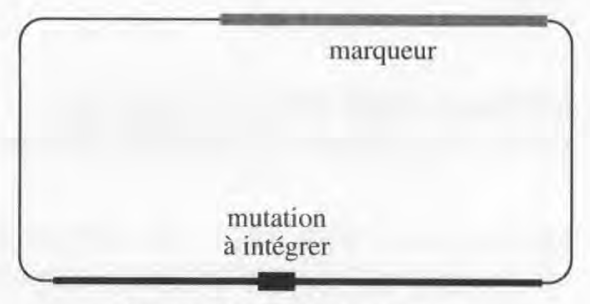

fragments d'ADN homologues

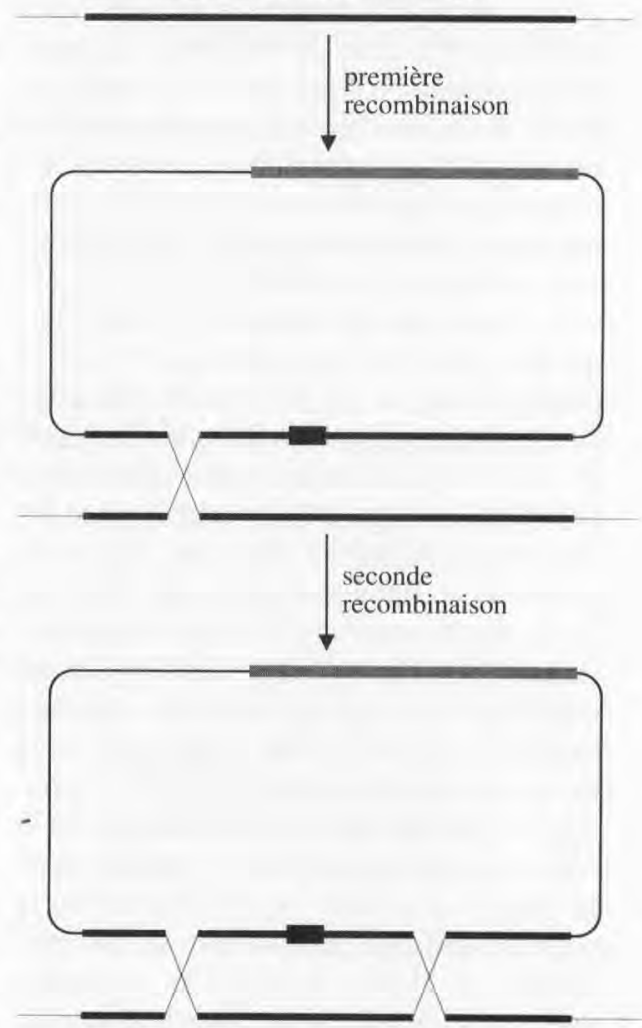




\section{POSSIBILITÉ DE CLONAGE PAR COMPLÉMENTATION DE MUTANT}

Le clonage d'un gène de Lactobacillus sake nécessaire pour la production de la sakacine $A$ et l'immunité envers cette bactériocine permet à Axelsson et al (1993) de montrer qu'il est possible de ne pas utiliser $E$ coli comme hôte. Leur travail était facilité par le fait que les gènes de la sakacine sont plasmidiques et qu'il existe un variant non producteur et sensible et présentant une bonne efficacité de transformation. L'avantage considérable de cette situation est qu'elle permet de cloner des gènes sans équivalent dans d'autres microorganismes par complémentation de mutants de la même espèce donc a priori sans problème d'expression.

\section{CONSTRUCTION DE MUTANTS}

Presque toutes les constructions de mutants publiées jusqu'à présent utilisent la technique classique d'intégration-excision par recombinaison homologue (voir fig 1). Dans un premier temps il faut obtenir dans un vecteur plasmidique le fragment d'ADN que l'on veut modifier puis effectuer la modification (mutation ponctuelle, délétion, insertion d'un gène hétérologue), introduire cette construction dans la souche à muter, attendre l'intégration de la totalité du plasmide modifié dans le chromosome, attendre l'excision des séquences plasmidiques et du fragment originel. Toutes les variations autour de ce thème permettent de pallier les difficultés rencontrées (mauvaise efficacité de transformation de la souche à modifier, impossibilité de sélectionner ou de cribler les phénotypes désirés). L'avantage considérable de ces techniques est que l'on peut obtenir des mutants parfaitement définis, stables, avec un dosage génique connu et fixe, sans trace de séquences provenant des vecteurs ou des marqueurs utilisés au cours de la construction ; on peut également obtenir des souches comportant plusieurs mutations ; il n'est pas nécessaire d'avoir une bonne efficacité de transformation. Leur limitation majeure est la nécessité de cloner au préalable le gène à modifier.

Lactobacillus plantarum est très utilisé comme inoculant d'ensilage; la quantité de sucres fermentescibles est souvent trop faible pour permettre une acidification suffisante ; on peut ajouter de la mélasse, du petit-lait ou de l'amidon avec de l' $\alpha$-amylase ; on peut aussi mieux utiliser le substrat végétal en ajoutant des cellulase, hémicellulase et pectinase. Une autre possibilité moins coûteuse serait de faire exprimer ces enzymes par $L$ plantarum. Scheirlinck et al (1989) ont montré que les gènes de l' $\alpha$ amylase de Bacillus stearothermophilus et de l'endoglucanase de Clostridium thermocellum s'expriment avec leurs propres promoteurs dans $L$ plantarum. Pour montrer la possibilité d'intégration dans le chromosome de $L$ plantarum ils ont d'abord inséré un fragment de l'ADN génomique de $L$ plantarum dans un vecteur d'E coli qui ne se réplique pas dans $L$ plantarum et qui comporte un gène de résistance à l'érythromycine. La transformation (avec une grande efficacité) de $L$ plantarum et la sélection par la résistance à l'érythromycine conduit à l'insertion, par recombinaison homologue, du plasmide au locus chromosomique d'où provient le fragment. Certaines des souches ainsi construites présentent une grande stabilité ségrégationnelle et des propriétés de croissance et de fermentation identiques à la souche d'origine. La même expérience avec les gènes de l' $\alpha$-amylase et de l'endoglucanase insérés dans le même plasmide conduit aux mêmes résultats. La fréquence d'intégration ne dépend pas de la longueur de la zone homologue dans la gamme de 1 à $8 \mathrm{~kb}$. L'intégration se fait très majoritairement dans la région d'homologie. L'activité de l'endoglucanase (parfaitement excrétée) 
est deux fois moins importante lorsque le gène est sur le chromosome (par rapport à une localisation plasmidique) ; par contre l'activité de l' $\alpha$-amylase est très faible. Une amplification en tandem de l'unité intégrée peut être obtenue par sélection pour une meilleure résistance à l'antibiotique marqueur. Une excision par recombinaison homologue peut être obtenue à basse fréquence lorsque l'on ne soumet plus la souche à la pression de sélection. Cette technique ne peut être mise en œuvre que si l'on dispose d'une grande efficacité de transformation puisque le plasmide ne se réplique pas dans la cellule hôte.

Bhowmik et Steele (1993) développent une procédure d'électroporation pour Lactobacillus helveticus suffisamment efficace pour permettre l'intégration d'un plasmide non réplicatif par recombinaison homologue au locus pepXP et obtenir ainsi une souche déficiente en X-prolyl-dipeptidyl-aminopeptidase. Le maintien dans un milieu non sélectif pour le marqueur permet la perte des séquences plasmidiques et le retour au phénotype sauvage.

Leer et al (1993) interrompent le gène cbh (conjugated bile salt hydrolase) de L plantarum par un plasmide non réplicatif comportant un gène de résistance à l'érythromycine et le gène $c b h$ interrompu par un gène de résistance au chloramphénicol. Bonne efficacité de transformation, sélection sur chloramphénicol : $90 \%$ sont résistants à l'érythromycine (intégration du plasmide entier); les $10 \%$ sensibles à l'érythromycine résultent vraisemblablement d'une double recombinaison. Dans ces derniers la résistance au chloramphénicol est parfaitement stable et l'activité Cbh est perdue.

La technique du vecteur suicide (non réplicatif) n'est pas adaptée lorsque la transformation de la souche hôte est peu efficace, ce qui est généralement le cas pour Lactobacillus plantarum. Rixon et al (1990) ont donc utilisé le plasmide pSA3 (navette
Streptococcus/E coli) et l'un de ses dérivés comprenant un fragment de $2,3 \mathrm{~kb}$ du chromosome de $L$ plantarum. Les rares transformants obtenus sont très instables en absence de pression de sélection. On peut néanmoins trouver dans une population non soumise à sélection quelques clones dont la résistance aux antibiotiques marqueurs est stable : ils correspondent à des événements d'intégration du plasmide dans le chromosome, par recombinaison homologue dans le cas du dérive comportant un fragment de chromosome. L'avantage de ce système est que l'on peut obtenir des événements d'intégration dans une population dérivant d'un seul transformant.

Une autre façon de pallier la faible efficacité de transformation est d'utiliser un plasmide à réplication thermosensible : á partir d'un seul transformant on peut établir, à basse température, une grande population comportant le plasmide de façon stable ; une augmentation de la température empêche la réplication du plasmide ; si la pression de sélection est maintenue sur un marqueur plasmidique, les seuls survivants résulteront d'un événement d'intégration. Maguin et al (1992) ont isolé un mutant à réplication thermosensible (pVE6002) à partir du plasmide pGK12 (dérivé de pWV01). Chez Lactococcus lactis la réplication de pVE6002 est possible à 28 ou $30^{\circ} \mathrm{C}$ et totalement impossible à partir de $35^{\circ} \mathrm{C}$. pVE6002 se comporte de la même façon chez Bacillus subtilis et Escherichia coli. La thermosensibilité est due à une mutation dans la protéine Rep. Biswas et al (1993) montrent que des dérivés de ce plasmide sont utilisables pour réaliser des intégrations (à haute température) puis des excisions (à basse température la réplication du plasmide a lieu et conduit à des recombinaisons) qui restaurent la structure chromosomique de départ ou qui aboutissent au remplacement par la structure portée par le plasmide. L'excision est tellement efficace qu'il n'est pas nécessaire de sélec- 
tionner les recombinants, ce qui ouvre la voie à la construction de souches sans phénotype facilement sélectionnable.

Les vecteurs dérivés de pVE6002 ne peuvent pas être utilisès chez les espèces (Lactobacillus helveticus par exemple) qui poussent très mal en dessous de $35^{\circ} \mathrm{C}$. Par contre le plasmide pSA3 (navette Streptococcus/E coli) est relativement stable à $37^{\circ} \mathrm{C}$ mais instable à $43^{\circ} \mathrm{C}$ chez $L$ helveticus. Bhowmik et al (1993) ont cloné un fragment interne du gène pepXP (X-prolyl-dipeptidyl-aminopeptidase) de $L$ helveticus dans pSA3. Une population de bactéries transformées par ce plasmide recombinant a été maintenue durant 30 générations à $45^{\circ} \mathrm{C}$ en présence d'érythromycine; toutes les colonies résultantes résistantes à l'érythromycine étaient déficientes pour la peptidase ; le plasmide avait été intégré au chromosome par recombinaison homologue. Lorsque l'on maintient une population résultant de cette intégration à $37^{\circ} \mathrm{C}$ (température permissive pour la réplication du plasmide) en l'absence d'érythromycine, on observe une perte de la résistance à l'érythromycine, un retour à l'activité peptidase et la disparition du fragment intégré par recombinaison homologue. Les auteurs ont donc poursuivi cette étude en utilisant le gène $p e p X P$ en partie délété. En deux étapes (intégration chromosomique par recombinaison homologue d'un côté de la délétion puis excision par recombinaison homologue de l'autre côté de la délétion) était construite une souche isogénique qui ne contenait plus trace de marqueur ou de vecteur.

Avec cette technique, Bhowmik et Steele (1994) ont pu interrompre le gène de la $D(-)$ lactate déshydrogénase de $L$ helveticus sans perturber la croissance ni la production totale d'acide lactique. Cette nouvelle souche produit donc deux fois plus d'acide $\mathrm{L}(+)$-lactique.

Pour étudier le rôle de chaque isomère de lactate chez Lactobacillus plantarum,
Ferain et al (1994) construisent une souche surproductrice de $\mathrm{L}-(+)$-lactate déshydrogénase et une souche délétée du gène correspondant (/dhL). L'efficacité de transformation n'étant pas suffisante cette délétion est construite par recombinaison homologue avec un plasmide à ségrégation instable (Hols et al, 1992) comportant une délétion d'un fragment interne du gène $I d h L$ et un marqueur de résistance. La souche transformée est maintenue 50 générations sans pression de sélection pour perdre les plasmides non intégrés ; les clones résistants (environ $1 \%$ ) sont sélectionnés et comportent le plasmide entier intégré au locus $/ d h L$; l'un de ces clones est maintenu 30 générations sans sélection; les clones sensibles (environ $1 \%$ ) sont criblés et ont perdu les séquences plasmidiques; la moitié de ces clones possède le gène $/ d h L$ intact, l'autre moitié le gène délété. La quantité totale d'acide lactique produit par un mutant de délétion est la même que celle du sauvage mais uniquement sous la forme $D$.

Un système similaire a été utilisé par Hols et al (1994) pour placer le gène de l' $\alpha$ amylase de Bacillus licheniformis au locus cbh de Lactobacillus plantarum en obtenant un niveau d'expression équivalent à la version plasmidique.

Pour pallier la faible sécrétion des amylases de Bacillus par L plantarum, Fitzsimons et al (1994) intègrent le gène de l'alphaamylase de Lactobacillus amylovorus au locus cbh de $L$ plantarum dont l'activité amylase extracellulaire est alors la moitié de celle de $L$ amylovorus. La souche modifiée croît mieux que la souche sauvage en présence d'amidon.

\section{INTÉGRATION SPÉCIFIQUE À UN SITE D'ATTACHEMENT DE PHAGE}

On peut utiliser les fonctions et les sites d'intégration spécifiques des phages pour, en une seule étape, introduire un fragment 
d'ADN. Cette technique suppose l'utilisation d'un plasmide non réplicatif donc la disponibilité d'une transformation efficace.

Raya et al (1992) construisent le plasmide PTRK182 en y insérant un fragment qui contient le site d'attachement attP et les fonctions nécessaires à l'intégration du phage tempéré $\varphi$ adh ce qui permet son intégration spécifiquement au site attB du chromosome de Lactobacillus gasseri. Nouveaux avantages de cette technique: introduction d'une seule copie d'un fragment d'ADN (homologue ou hétérologue) ; ce fragment peut être de grande taille (le génome du phage padh a une longueur de $43 \mathrm{~kb}$ ); pas d'effet sur la viabilité puisque le site d'intégration n'est pas essentiel.

Le phage tempéré mv4 peut lysogéniser plusieurs souches de Lactobacillus delbrueckii. Dupont et al (1995) ont montré que le site d'attachement attB est à la fin du gène d'un tRNASer. Ils ont pu construire un vecteur non réplicatif comportant le site d'attachement attP et le gène de l'intégrase et montrer que ce plasmide s'intègre dans le génome d'un autre lactobacille ( $L$ plantarum) spécifiquement à la fin du tRNASer équivalent qui semble rester parfaitement fonctionnel. Sous réserve que les séquences plasmidiques insérées ne présentent pas de problème d'expression, ce systeme permettrait de construire une souche en partie diploïde donc d'ouvrir la voie à des études d'interaction entre allèles.

L'utilisation des vecteurs d'intégration basés sur la recombinaison homologue est limitée au régions préalablement clonées; les vecteurs basés sur les sites d'attachement attP sont limités par l'existence d'un site attB correspondant. Walker et Klaenhammer (1994) ont construit un vecteur suicide autour d'un élément d'insertion, I'IS 1223 de Lactobacillus johnsonii. Ce vecteur peut s'intégrer à quatre positions différentes dans le chromosome de $L$ johnsonii (recombinaison homologue avec les IS déjà présents) et de façon aléatoire dans le chro- mosome de Lactobacillus gasseri (grâce aux fonctions de transposition de l'IS), permettant ainsi la mutagenèse par insertion, cartographie ou intégration d'éléments hétérologues.

\section{CONCLUSION}

L'ensemble des données présentées cidessus montrent clairement la grande utilité des mutants déjà obtenus dans les genres Lactobacillus, Leuconostoc et Pediococcus, que ce soit pour analyser des systèmes connus chez d'autres microorganismes ou pour comprendre des caractéristiques particulières aux bactéries lactiques. L'impossibilité (provisoire?) de transférer par les moyens classiques et rapides (transformation, transduction, conjugaison) des mutations d'une souche à l'autre limite les possibilités d'analyse des interactions entre allèles ou entre gènes et impose de faire des mutagenèses suffisamment douces pour éviter l'obtention de doubles mutants aux phénotypes incompréhensibles.

La possibilité d'intégrer dans le chromosome de toutes les souches transformables (même avec une faible efficacité) toutes les structures désirées ouvre la voie, d'une part, à l'analyse précise du rôle et du fonctionnement de chaque gène et, d'autre part, à la construction de souches améliorées dans un but économique. Ces modifications sont actuellement limitées par l'étape de clonage de l'ADN correspondant.

On doit donc envisager de cloner certains gènes sans équivalent dans d'autres microorganismes par complémentation directe de mutants obtenus par les méthodes classiques. II deviendrait ainsi possible de pallier l'absence des techniques classiques de transfert de gènes (avec les moyens de la biologie moléculaire, certes plus lourds) et de bénéficier néanmoins de la puissance de l'analyse génétique. 


\section{RÉFÉRENCES}

Atlan D, Laloi P, Portalier R (1989) Isolation and characterization of aminopeptidase-deficient Lactobacillus bulgaricus mutants. Appl Environ Microbiol 55. $1717-1723$

Atlan D, Laloi P, Portalier R (1990) X-prolyl-dipeptidy aminopeptidase of Lactobacillus delbrueckil subsp bulgaricus : characterization of the enzyme and isolation of deficient mutants. Appl Environ Microbiol $56,2174-2179$

Axelsson L, Holck A, Birkeland SE, Aukrust T, Blom H (1993) Cloning and nucleotide sequence of a gene from Lactobacillus sake Lb706 necessary for sakacin A production and immunity $\mathrm{Appl}$ Environ Microbiol 59, 2868-2875

Bhowmik T, Steele JL (1993) Development of an electroporation procedure for gene disruption in Lactobacillus helveticus CNRZ 32. J Gen Microbiol 139, 1433-1439

Bhowmik T, Steele JL (1994) Cloning, characterization and insertional inactivation of the Lactobacillus helveticus $\mathrm{D}(-)$ lactate dehydrogenase gene. Appl Microbiol Biotechnol 41, 432-439

Bhowmik T, Fernández L, Steele JL (1993) Gene replacement in Lactobacillus helveticus. J Bacteriol 175 , 6341-6344

Biswas I, Gruss A, Ehrlich SD, Maguin E (1993) High-efficiency gene inactivation and replacement system for Gram-positive bacteria. J Bacteriol 175, 36283635

Cavin JF, Prévost H, Lin J, Schmitt P, Diviès C (1989) Medium for screening Leuconostoc oenos strains defective in malolactic fermentation. Appl Environ Microbiol 55, 751-753

Chagnaud P, Naouri P, Arnaud A, Galzy P (1990) A characterization method for mutants in malolactic enzyme activity. J Microbiol Meth 11, 213-218

Daeschel MA, McFeeters RF, Fleming HP, Klaenhammer TR, Sanozky RB (1984) Mutation and selection of Lactobacillus plantarum strains that do not produce carbon dioxide from malate. Appl Environ Microbiol $47,419-420$

Deguchi Y, Morishita T (1992) Nutritional requirements in multiple auxotrophic lactic acid bacteria : genetic lesions affecting amino acid biosynthetic pathways in Lactococcus lactis, Enterococcus faecium, and Pediococcus acidilactici. Biosci Biotechnol Biochem $56,913-918$

Dupont L, Boizet-Bonhoure B, Coddeville M, Auvray F. Ritzenthaler $P$ (1995) Characterization of genetic elements required for site-specific integration of Lactobacillus delbrueckil subsp bulgaricus bacteriophage mv4 and construction of an integration-proficient vector for Lactobacillus plantarum. J Bacteriol $177,586-595$
Ferain T, Garmyn D, Bernard N, Hols P, Delcour J (1994) Lactobacillus plantarum IdhL gene : overexpression and deletion. J Bacteriol 176, 596-601

Fitzsimons A, Hols P, Jore J, Leer RJ, O'Connell M, Delcour J (1994) Development of an amylolytic Lactobacillus plantarum silage strain expressing the Lactobacillus amylovorus $\alpha$-amylase gene. Appl Environ Microbiol 60, 3529-3535

Hols P, de Halleux S, Delcour J (1992) A strategy to construct vector-free amylolytic strains through nondisruptive homologous recombination: application to Enterococcus faecalis. Gene 118, 31-38

Hols P, Ferain T, Garmyn D, Bernard N, Delcour J (1994) Use of homologous expression-secretion signals and vector-free stable chromosomal integration in engineering of Lactobacillus plantarum for $\alpha$-amylase and levanase expression. Appl Environ Microbiol $60,1401-1413$

Kim D, Robyt JF (1994) Production and selection of mutants of Leuconostoc mesenteroides constitutive for glucansucrases. Enzyme Microb Technol 16, 659-664

Kim D, Robyt JF (1995) Production, selection, and characteristics of mutants of Leuconostoc mesenteroides B-742 constitutive for dextransucrases. Enzyme Microb Technol 17, 689-695

Lautensach A, Subden RE (1987) Isolation and characterization of Leuconostoc oenos mutants with defective malo-lactic enzyme. Microbios 52, 115-127

Leathers TD, Hayman GT, Cote GL (1995) Rapid screening of Leuconostoc mesenteroides mutants for elevated proportions of alternan to dextran. Curr Microbiol 31, 19-22

Leer RJ, Christiaens H, Verstraete W, Peters L, Posno M, Pouwels PH (1993) Gene disruption in Lactobacillus plantarum strain 80 by site-specific recombination: isolation of a mutant strain deficient in conjugated bile salt hydrolase activity. Mol Gen Genet 239, 269-272

Maguin E, Duwat P, Hege T, Ehrlich D, Gruss A (1992) New thermosensitive plasmid for Gram-positive bacteria. J Bacteriol 174, 5633-5638

Masson A (1992) Étude génétique, physiologique et biochimique du métabolisme des pyrimidines chez Lactobacillus plantarum. Thèse de l'université LouisPasteur, Strasbourg

Masson A, Kammerer B, Hubert JC (1994) Selection and biochemical studies of pyrimidine-requiring mutants of Lactobacillus plantarum. J Appl Bacteriol 77, 88-95

Mizutani N, Yamada M, Takayama K, Shoda M (1994) Constitutive mutants for dextransucrase from $\mathrm{Leu}$ conostoc mesenteroides NRRL B-512F. J Ferment Bioeng 77, 248-251

Mollet B, Delley M (1990) Spontaneous deletion formation within the $\beta$-galactosidase gene of Lactobacillus bulgaricus. J Bacteriol 172, 5670-5676 
Mollet B, Delley M (1991) A $\beta$-galactosidase deletion mutant of Lactobacillus bulgaricus reverts to generate an active enzyme by internal DNA sequence duplication. Mol Gen Genet 227, 17-21

Morishita T, Yura T (1976) Altered nutritional requirements associated with mutations affecting the structure of ribonucleic acid polymerase in Lactobacillus casei. J Bacteriol 125, 416-422

Morishita T, Yajima M (1995) Incomplete operation of biosynthetic and bioenergetic functions of the citric acid cycle in multiple auxotrophic lactobacilli. Biosci Biotechnol Biochem 59, 251-255

Morishita T, Fukada T, Shirota M, Yura T (1974) Genetic basis of nutritional requirements in Lactobacillus casei. J Bacteriol 120, 1078-1084

Morishita T, Deguchi Y, Yajima M, Sakurai T, Yura T (1981) Multiple nutritional requirements of lactobacilli : genetic lesions affecting amino acid biosynthetic pathways. J Bacteriol 148, 6471

Ntamere AS, Taron DJ, Neuhaus FC (1987) Assembly of D-alanyl-lipoteichoic acid in Lactobacillus casei: mutants deficient in the D-alanyl ester content of this amphiphile. J Bacteriol 169, 1702-1711

Raya RR, Fremaux C, de Antoni GL, Klaenhammer TR (1992) Site-specific integration of the temperate bacteriophage $\varphi$ adh into the Lactobacillus gasserichromosome and molecular characterization of the phage (attP) and bacterial (attB) attachment sites. J Bacteriol 174, 5584-5592

Recsei PA, Snell EE (1972) Histidine decarboxylaseless mutants of Lactobacillus 30a: isolation and growth properties. J Bacteriol 112, 624-626

Renault PP, Heslot H (1987) Selection of Streptococcus lactis mutants defective in malolactic fermentation. Appl Environ Microbiol 53, 320-324

Rixon JE, Hazlewood GP, Gilbert HJ (1990) Integration of an unstable plasmid into the chromosome of Lactobacillus plantarum. FEMS Microbiol Lett 71, 105-109
Rodriquez-Quinones (sic) F, Megias M, Palomares AJ, Ruiz-Berraquero F (1982) Nitrosoguanidine mutagenesis in Lactobacillus plantarum. Microbios Lett $20,75-80$

Rodríguez-Quiñones F, Palomares AJ, Megias M, RuizBerraquero $F$ (1984) The influence of several variables for nitrosoguanidine mutagenesis in Lactobacillus plantarum. Curr Microbiol 10, 137-140

Scheirlinck T, Mahillon J, Joos H, Dhaese P, Michiels $F(1989)$ Integration and expression of $\alpha$-amylase and endoglucanase genes in the Lactobacillus plantarum chromosome. Appl Environ Microbiol 55, 21302137

Shimizu-Kadota M, Sakurai T (1982) Prophage curing in Lactobacillus caseiby isolation of a thermoinducible mutant. Appl Environ Microbiol 43, 1284-1287

Smith MR, Zahnley J, Goodman N (1994) Glucosyltransferase mutants of Leuconostoc mesenteroides NRRL B-1355. Appl Environ Microbiol 60, 2723-2731

Tamura C, Matsushita O (1992) Melibiose transport system in Lactobacillus plantarum. Microbiol Immunol 36 , 1119-1128

Veyrat A, Monedero V, Pérez-Martínez G (1994) Glucose transport by the phosphoenolpyruvate:mannose phosphotransferase system in Lactobacillus casei ATCC 393 and its role in carbon catabolite repression. Microbiology 140, 1141-1149

Walker DC, Klaenhammer TR (1994) Isolation of a novel IS3 group insertion element and construction of an integration vector for Lactobacillus spp. J Bacteriol $176,5330-5340$

Widdowson D, White PJ (1976) The nutritional requirements of methicillin-dependent and -resistant strains of Pediococcus cerevisiae. J Gen Microbiol 92, 138146

Zúñiga M, Ferrer S, Pardo I (1994) A selective medium for the isolation of malolactic mutants of Leuconostoc oenos, Lett Appl Microbiol 19, 451-453 